\title{
A three-dimensional metal-organic framework with a distorted Kagome related layer showing canted antiferromagnetic behaviour $\dagger$
}

\author{
Partha Mahata, ${ }^{a}$ Diptiman Sen $* b$ and Srinivasan Natarajan $* a$ \\ Received (in Cambridge, UK) 5th October 2007, Accepted 14th November 2007 \\ First published as an Advance Article on the web 22nd November 2007 \\ DOI: $10.1039 / b 715314 g$
}

Three-dimensional $\left[\mathrm{Mn}_{3}\left\{\mathrm{C}_{6} \mathrm{H}_{3}(\mathrm{COO})_{3}\right\}_{2}\right]$ comprising twodimensional -Mn-O-Mn- distorted Kagome layers, formed by two geometrically different hexa-coordinated $\mathrm{Mn}^{2+}$ ions, show less frustration and spin canted long range ordering possibly due to antisymmetric exchange.

Metal-organic frameworks (MOFs) based on transition metal elements are an important family as they provide an opportunity to correlate their structure and magnetism, in addition to their adsorption and related properties arising out of the porous structure. ${ }^{1}$ Recently, two-dimensional (2D) spin-half antiferromagnets have generated considerable interest due to the observation of superconductivity and spin chirality. ${ }^{2}$ Among the $2 \mathrm{D}$ lattices, ${ }^{2 b}$ the Kagome lattice, possessing either 3- or 6-fold symmetry within the layers, occupies an important position for the observation of magnetic frustration. Most of the magnetic studies of Kagome structured materials are with jarosite, $\mathrm{KFe}_{3}\left(\mathrm{SO}_{4}\right)_{2}(\mathrm{OH})_{6}$, and its analogues. ${ }^{3}$ Recently Rao and Nocera and co-workers independently studied various transition metal based jarosite related compounds with particular emphasis on the magnetic properties of Kagome structures. ${ }^{4,5}$ Zaworotko and co-workers reported the first crystal engineered Kagome lattice whose lattice vertexes are occupied by paramagnetic dicopper(II) tetracarboxylate spin pairs. ${ }^{6}$ The $\mathrm{Cu}^{2+}$ ions within the dimer are coupled antiferromagnetically, but the bridges between the dimers are too long to allow effective magnetic coupling. Recently Gao and co-workers prepared a $\mathrm{Co}^{2+}$ compound, $\mathrm{Co}\left(\mathrm{N}_{3}\right)_{2}(\mathrm{bpg})(\mathrm{DMF})_{4 / 3}$, with a Kagome lattice, which shows both frustration and long range ordering. ${ }^{7}$ Kagome-like topologies, though few, have been observed in other MOFs and related systems as well. ${ }^{8}$ In jarosite structures, the metal centers are connected through a single atom $(\mathrm{O}, \mathrm{F}, \mathrm{OH}$ etc. $),{ }^{4,5}$ but there are no reports of similar layers in MOFs. Recently, we have reported the formation of a brucite related structure, $[\mathrm{Mn}(\mathrm{OH})]_{2}\left[\mathrm{C}_{12} \mathrm{H}_{8} \mathrm{O}-\right.$ $\left.(\mathrm{COO})_{2}\right]$ where the metal centers are bridged by a single atom $(\mathrm{O}, \mathrm{OH}) .{ }^{9}$ Continuing a similar strategy and employing trimellitic acid (1,2,4-benzene tricarboxylic acid, BTC) along with $\mathrm{Mn}^{2+}$ ions, we have isolated a unique solid possessing

\footnotetext{
${ }^{a}$ Framework Solids Laboratory, Solid State and Structural Chemistry Unit, Indian Institute of Science, Bangalore 560 012, India. Fax: +91-80-2360-1310.E-mail: snatarajan@sscu.iisc.ernet.in

${ }^{b}$ Centre for High Energy Physics, Indian Institute of Science, Bangalore 560 012, India.E-mail:diptiman@cts.iisc.ernet.in $\dagger$ Electronic supplementary information (ESI) available: X-Ray crystallographic data, XRD pattern, IR, EPR, TGA, magnetic studies and additional figures. See DOI: 10.1039/b715314g
}

a distorted Kagome layer in which the metal centers are connected by a single atom $(\mathrm{O})$. The three-dimensional compound $\left[\mathrm{Mn}_{3}\left\{\mathrm{C}_{6} \mathrm{H}_{3}(\mathrm{COO})_{3}\right\}_{2}\right]$, I, has distorted Kagome layers formed by two geometrically different $\mathrm{Mn}^{2+}$ ions, connected by the trimellitate anions. This is the first observation, to our knowledge, of the metal ions being connected by a single bridging atom to form a distorted Kagome layer in MOFs. In this communication, we present the synthesis, structure and magnetic behavior of $\mathbf{I} . \ddagger$

The asymmetric unit of $\mathbf{I}$ consists of two independent $\mathrm{Mn}^{2+}$ ions and one trimellitate anion. While $\mathrm{Mn}(1)$ is in a general position, $\mathrm{Mn}(2)$ lies on an inversion centre with a site multiplicity of 0.5 . Though both of the $\mathrm{Mn}^{2+}$ ions are hexa coordinated, the $\mathrm{Mn}(1)$ has distorted trigonal prismatic geometry and $\mathrm{Mn}(2)$ has an octahedral geometry with carboxylate oxygen atoms (Fig. 1a). The $\mathrm{Mn}(2) \mathrm{O}_{6}$ octahedral units are connected with two $\mathrm{Mn}(1) \mathrm{O}_{6}$ trigonal prismatic units via two different $\mu_{3}$ carboxylate oxygen atoms $(\mathrm{O}(3)$ and $\mathrm{O}(4))$ and two $\mathrm{Mn}(1) \mathrm{O}_{6}$ units are connected via $\mathrm{O}(5) \mu_{3}$ carboxylate oxygen

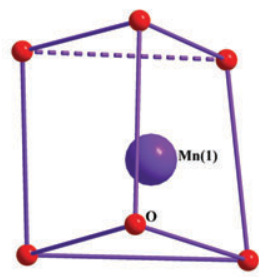

(i)

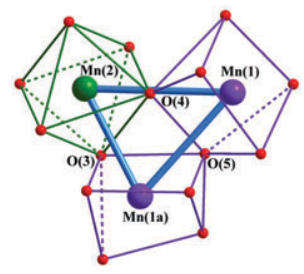

(b)

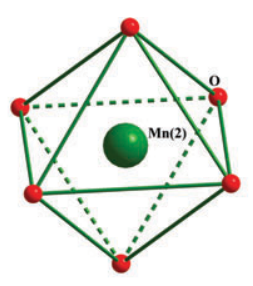

(ii)

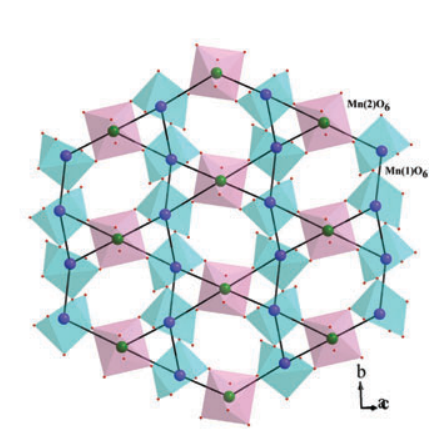

(c)

Fig. 1 (a) The octahedral (i) and trigonal prismatic geometry (ii) of $\mathrm{Mn}^{2+}$ ions in $\mathbf{I}$, (b) the structure of the triangular unit formed by the connectivity of two $\mathrm{Mn}(1)^{2+}$ and one $\mathrm{Mn}(2)^{2+}$ ions, and (c) a polyhedral view of the Kagome layer formed by the connectivity of $\mathrm{Mn}(1) \mathrm{O}_{6}$ and $\mathrm{Mn}(2) \mathrm{O}_{6}$ polyhedral units. 


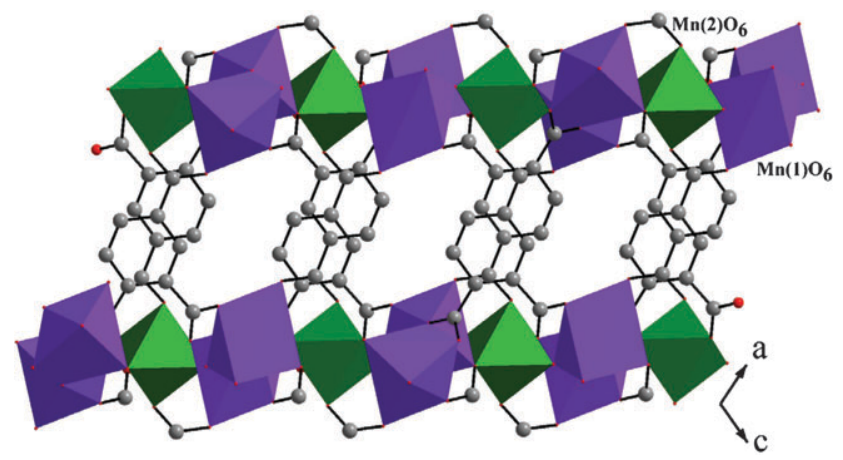

Fig. 2 The connectivity between the Kagome layers in I through 1,2,4-BTC units.

atom ( $\mu_{3}-\mathrm{O}$ connects one carbon and two $\mathrm{Mn}$ ) forming a triangular lattice (Fig. 1b), which are connected through the corners to form a layer that resembles the Kagome arrangement (Fig. 1c). Topologically, the distorted Kagome layer can be considered as a 4-connected node with $3^{2} 6^{2}$ net. A careful examination of the triangular arrangement of the metal ions in I indicates three distinct $\mathrm{Mn}-\mathrm{Mn}$ distances and $\mathrm{Mn}-\mathrm{O}-\mathrm{Mn}$ angles: $3.36,3.8$ and $4.1 \AA$; 97.88, 130.53 and $112.28^{\circ}$. The $\mathrm{Mn}-\mathrm{Mn}$ connectivity within the layer shows that each of the triangles within the layer is formed by two $\mathrm{Mn}(1)$ and one $\mathrm{Mn}(2)$ species (Fig. 1c). The benzene carboxylate units connect the layers three-dimensionally (Fig. 2).

The magnetic studies carried out on $\mathbf{I}$ are shown in Fig. 3. At RT, the effective magnetic moment $\left(\mu_{\text {eff }}\right)$ is $6.01 \mu_{\mathrm{B}}$. The $\chi_{\mathrm{M}} T$ versus $T$ curve is shown in Fig. 3a. The $\chi_{\mathrm{M}} T$ value decreases continuously with decreasing temperature and reaches a minimum of $2.59 \mathrm{emu} \mathrm{mol}^{-1} \mathrm{~K}$ at $23 \mathrm{~K}$. On cooling further $\chi_{\mathrm{M}} T$ increases rapidly suggesting that the magnetic behavior at low temperature should be either ferrimagnetic or canted antiferromagnetic. ${ }^{10}$ The $1 / \chi_{M}$ vs. $T$ curve is shown as the inset of Fig. 3a. Above $100 \mathrm{~K}$, the magnetic behavior can be fitted by the Curie-Weiss law, with $C=5.35 \mathrm{emu} \mathrm{mol}^{-1}$ and $\theta_{\mathrm{P}}=-46.1 \mathrm{~K}$. The negative $\theta_{\mathrm{P}}$ indicates antiferromagnetic coupling between the $\mathrm{Mn}^{+2}$ ions. The field-cooled (FC) and zero-field-cooled (ZFC) magnetizations measured at 50 Oe down to $2 \mathrm{~K}$, indicate a deviation at $13 \mathrm{~K}$. AC magnetic susceptibility studies indicate (see ESI $\dagger$ ) a sharp peak suggesting a transition with a critical temperature $T_{\mathrm{c}} \approx 13 \mathrm{~K}$. The degree of frustration, $f$, defined as $\theta_{\mathrm{P}} / T_{\mathrm{c}}{ }^{11}$ was found to be 3.55 , which is far lower than that observed in the classical Kagome lattice of iron jarosite. ${ }^{12}$ The field-cooled $\chi_{\mathrm{M}} T$ values at different applied fields as a function of temperature show a spontaneous increase around $13 \mathrm{~K}$, which is more noticeable for lower field strengths (Fig. 3c). The drop in $\chi_{\mathrm{M}} T$ below $13 \mathrm{~K}$ may be due to the magnetic field saturation effect. ${ }^{10}$ The field dependence of the magnetization, $M$ vs. $H(0-50 \mathrm{kOe})$ at $5 \mathrm{~K}$ (Fig. 3d), shows behavior typical of a canted antiferromagnet with a sharp increase of the magnetization at low fields and a linear variation of $M$ at $H>20$ kOe. ${ }^{13}$ At $50 \mathrm{kOe}$ the magnetization value reaches $1.57 \mu_{\mathrm{B}}$, a value that is far below the saturation value of $5.92 \mu_{\mathrm{B}}$ expected for a spin-only $\mathrm{Mn}^{2+}$ ion. This lack of saturation at high field confirms that the low temperature magnetic ground state is a

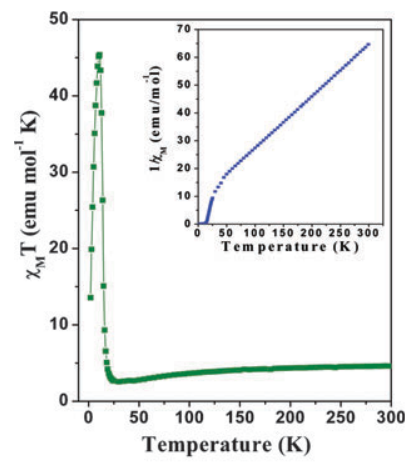

(a)

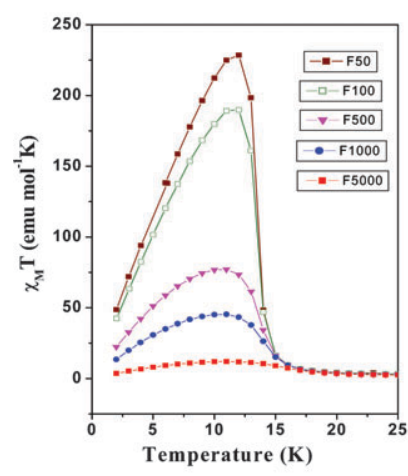

(c)

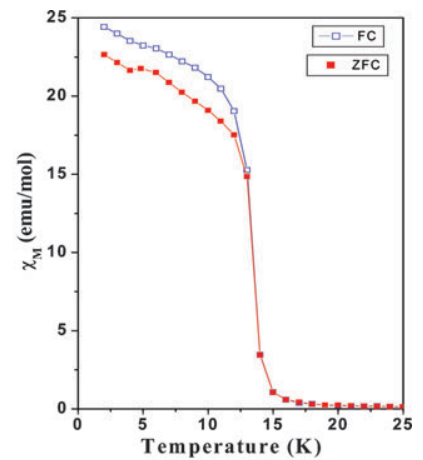

(b)

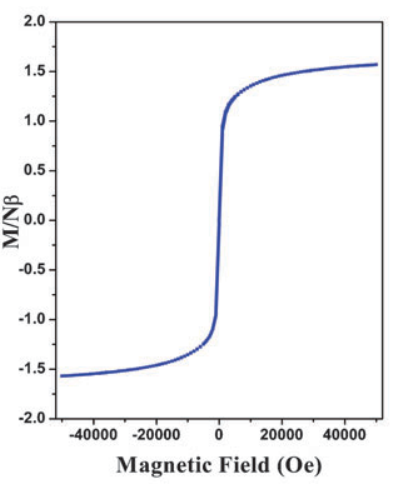

(d)
Fig. 3 (a) Plot of $\chi_{\mathrm{M}} T$ vs. $T(H=1000$ Oe) (FC). Inset shows the $1 / \chi_{\mathrm{M}}$ vs. $T$ plot, (b) field cooled (FC) and zero-field cooled (ZFC) plot of $\chi_{\mathrm{M}} v s . T$ at $H=50 \mathrm{Oe}$, (c) $\chi_{\mathrm{M}} T v s . T$ plot at various fields, (d) $M v s$. $H$ plot at $5 \mathrm{~K}$

canted antiferromagnetic one, ${ }^{14}$ which is due to the spin canting caused by the antisymmetric Dzyaloshinsky-Moriya (DM) exchange.

Taking into account the structural features of this compound, further inspection of the magnetic behavior is worthwhile to understand the smaller frustration and the origin of the DM interaction. The recent reports of $\mathrm{Mn}^{2+}$ carboxylates, $\left[\mathrm{Mn}\left\{\mathrm{Mn}_{3}\left(\mu_{3}-\mathrm{F}\right)(\mathrm{bta})_{3}\left(\mathrm{H}_{2} \mathrm{O}\right)_{6}\right\}\right]$ and $\left[\mathrm{Mn}_{2}(\mathrm{THFTC})\left(\mathrm{H}_{2} \mathrm{O}\right)_{3}\right]$, with triangular arrangements of the $\mathrm{Mn}^{2+}$ ions show no long range ordering. ${ }^{15}$ The manganese carboxylate, $\left[\mathrm{Mn}_{3}\left\{\mathrm{C}_{6} \mathrm{H}_{3}(\mathrm{COO})_{3}\right\}_{2}\right]$, with an isolated linear $\mathrm{Mn}_{3}$ unit connected through trimesate anions show simple antiferromagnetic behavior through intra- and inter-trimer interactions. ${ }^{16}$ In all these compounds $\mathrm{Mn}^{2+}$ ions are exclusively present in octahedral coordination. In the present compound, however, $\mathrm{Mn}^{2+}$ ions are in trigonal prismatic and octahedral geometry (Fig. 1a). Antiferromagnetic interactions between the Mn and $\mathrm{O}$ atom occurs through the super exchange process using metal $\mathrm{d}$ orbitals and oxygen $\mathrm{p}$ orbitals. The octahedral geometry $[\mathrm{Mn}(2)]$ is more favorable for overlap between the $\mathrm{p}$ orbitals of oxygens and $\mathrm{d}_{x^{2}-y^{2}}$ and $\mathrm{d}_{z^{2}}$ orbitals of the $\mathrm{Mn}^{2+}$ ions. In the trigonal prismatic geometry [Mn(1)], the overlap is between the $\mathrm{d}_{x z}$ and $\mathrm{d}_{y z}$ orbitals of $\mathrm{Mn}^{2+}$ and the $\mathrm{p}$ orbitals of oxygen. ${ }^{17}$ This difference in the super exchange pathway between the $\mathrm{Mn}^{2+}$ ions via the oxygens can give rise to a large antisymmetric DM interaction, which is responsible for the spin canting in our structure..$^{2 c, 3 d, 18}$ This geometric 
difference between the $\mathrm{Mn}^{2+}$ ions (in particular, the different $\mathrm{Mn}-\mathrm{Mn}$ distances and angles mentioned above) reduces the frustration within the triangular lattice and at the same time both the in-plane and out-of-plane DM vectors would be nonzero in this system, with the in-plane component inducing canted spin moments in the out-of-plane directions resulting in long range ordering in $\mathbf{I}$. The inter-layer distance in $\mathbf{I}$ is $\sim 8 \AA$, which suggests that the magnetic interactions are confined within the layers.

In summary, $\left[\mathrm{Mn}_{3}\left\{\mathrm{C}_{6} \mathrm{H}_{3}(\mathrm{COO})_{3}\right\}_{2}\right]$ represents first single atom bridged Kagome related layer in a three-dimensional MOF compound formed by two geometrically different $\mathrm{Mn}^{2+}$ species. The presence of the trigonal prismatic and octahedrally coordinated $\mathrm{Mn}^{2+}$ ions reduces the frustration giving rise to canted antiferromagnetism through antisymmetric Dzyaloshinsky-Moriya (DM) exchange.

Authors thank DST and CSIR, Government of India, for the award of research grants and fellowships [RAMANNA (DST), SRF (CSIR)].

\section{Notes and references}

$\ddagger$ A mixture of $\mathrm{Mn}(\mathrm{OAc})_{2} \cdot 4 \mathrm{H}_{2} \mathrm{O}:$ 1,2,4-tricarboxylic acid : imidazole $: \mathrm{KOH} / \mathrm{LiOH}:$ water $(1: 1: 1: 2: 444)$ was reacted in a PTFE-lined acid digestion vessel $(23 \mathrm{~mL})$ at $220{ }^{\circ} \mathrm{C}$ for $24 \mathrm{~h}$. Colorless rectangular crystals of $\mathbf{I}$ were collected (yield $\approx 70 \%$ ) after washing with a sufficient quantity of water and diethyl ether. The starting $\mathrm{pH}$ value and the $\mathrm{pH}$ value after the reaction were 6 and 4.5, respectively. Anal. Calcd for I: C, 37.30; H, 1.04. Found: C, 37.12; H, 1.11. The compound was characterized by IR, TGA and EPR spectroscopic studies. The structure was determined using a Bruker AXS smart Apex CCD diffractometer at 293(2) K. The structure was solved and refined using SHELXL97 present in the WinGx suite of programs (Version 1.63.04a). Crystal data for $\mathbf{I}:\left[\mathrm{Mn}_{3}\left\{\mathrm{C}_{6} \mathrm{H}_{3}(\mathrm{COO})_{3}\right\}_{2}\right], M=579.04$, monoclinic, space group $P 2_{1} / n$ (no. 14), $a=11.314(3), b=$ 6.5962(16), $c=11.391(3) \AA, \beta=111.029(4) ; V=793.5(3) \AA^{3}, Z$ $=2, \rho_{\mathrm{cal}}=2.424 \mathrm{~g} \mathrm{~cm}^{-3}, \mu\left(\mathrm{Mo}_{\mathrm{K} \alpha}\right)=2.435 \mathrm{~mm}^{-1}, 6663$ reflections, 1853 unique $\left(R_{\mathrm{int}}=0.03\right), 1600$ observed $I>2 \sigma(I), R_{1}=0.0425$, $\mathrm{w} R_{2}=0.0714$ and GOF $=1.089$ for 151 parameters. CCDC 637648 . For crystallographic data in CIF or other electronic format see DOI: $10.1039 / \mathrm{b} 715314 \mathrm{~g}$

1 (a) A. K. Cheetham, C. N. R. Rao and R. K. Feller, Chem. Commun., 2006, 4780; (b) D. Maspoch, D. Ruiz-Molina and J. Veciana, Chem. Soc. Rev., 2007, 36, 770.

2 (a) J. E. Greedan, J. Mater. Chem., 2001, 11, 37; (b) J. Richter, J. Schulenburg and A. Honecker, Quantum Magnetism, SpringerVerlag, Berlin, 2004; (c) D. Grohol, K. Matan, J. H. Cho, S. H.
Lee, J. W. Lynn, D. G. Nocera and Y. S. Lee, Nat. Mater., 2005, 4, 323.

3 (a) A. B. Harris, C. Kallin and A. J. Berlinsky, Phys. Rev. B: Condens. Matter Mater. Phys., 1992, 45, 2899; (b) T. Inami, M. Nishiyama, S. Maegawa and Y. Oka, Phys. Rev. B: Condens. Matter Mater. Phys., 2000, 61, 12181; (c) A. S. Wills, A. Harrison, C. Ritter and R. I. Smith, Phys. Rev. B: Condens. Matter Mater. Phys., 2000, 61, 6156; (d) M. Elhajal, B. Canals and C. Lacroix, Phys. Rev. B: Condens. Matter Mater. Phys., 2002, 66, 014422.

4 (a) J. N. Behera and C. N. R. Rao, J. Am. Chem. Soc., 2006, 128, 9334; (b) J. N. Behera and C. N. R. Rao, Dalton Trans., 2007, 669; (c) J. N. Behera, G. Paul, A. Choudhury and C. N. R. Rao, Chem. Commun., 2004, 456; (d) C. N. R. Rao, E. V. Sampathkumaran, R. Nagarajan, G. Paul, J. N. Behera and A. Choudhury, Chem. Mater., 2004, 16, 1441.

5 (a) B. M. Bartlett and D. G. Nocera, J. Am. Chem. Soc., 2005, 127, 8985; (b) D. Grohol, D. G. Nocera and D. Papoutsakis, Phys. Rev. B: Condens. Matter Mater. Phys., 2003, 67, 064401; (c) D. Papoutsakis, D. Grohol and D. G. Nocera, J. Am. Chem. Soc., 2002, 124, 2647; (d) D. G. Nocera, B. M. Bartlett, D. Grohol, D. Papoutsakis and M. P. Shores, Chem.-Eur. J., 2004, 10, 3850.

6 B. Moulton, J. Lu, R. Hajndl, S. Hariharan and M. J. Zaworotko, Angew. Chem., Int. Ed., 2002, 41, 2821.

7 X. Y. Wang, L. Wang, Z. M. Wang and S. Gao, J. Am. Chem. Soc., 2006, 128, 674.

8 (a) H. Chun and J. Moon, Inorg. Chem., 2007, 46, 4371; (b) E. B. Rusanov, V. V. Ponomarova, V. V. Komarchuk, H. StoeckliEvans, E. Fernandez-Ibanez, F. Stoeckli, J. Sieler and K. V. Domasevitch, Angew. Chem., Int. Ed., 2003, 42, 2499; (c) J. J. Perry, G. J. McManus and M. J. Zaworotko, Chem. Commun., 2004, 2534; (d) Y. Liu, V. Ch. Kravtsov, D. A. Beauchamp, J. F. Eubank and M. Eddaoudi, J. Am. Chem. Soc., 2005, 127, 7266; (e) C. J. Li, S. Hu, W. Li, C. K. Lam, Y. Z. Zheng and M. L. Tong, Eur. J. Inorg. Chem., 2006, 1931.

9 P. Mahata, A. Sundaresan and S. Natarajan, Chem. Commun., 2007, 4471.

10 M. Yang, J. Yu, L. Shi, P. Chen, G. Li, Y. Chen, R. Xu and S. Gao, Chem. Mater., 2006, 18, 476.

11 M. A. Girtu, C. M. Wynn, W. Fujita, K. Awaga and A. J. Epstein, Phys. Rev. B: Condens. Matter Mater. Phys., 1998, 57, R11058.

12 A. Harrison, J. Phys.: Condens. Matter, 2004, 16, S553.

13 M. Riou-Cavellec, C. Lesaint, M. Nogues, J. M. Greneche and G. Ferey, Inorg. Chem., 2003, 42, 5669.

14 (a) R. L. Carlin, Magnetochemistry, Springer-Verlag, Berlin, 1986; (b) F. Sanz, C. Parada, J. M. Rojo and C. Ruiz-Valero, Chem. Mater., 2001, 13, 1334.

15 (a) E. Q. Gao, N. Liu, A. L. Cheng and S. Gao, Chem. Commun., 2007, 2470; (b) X. Y. Wang and S. C. Sevov, Chem. Mater., 2007, 19, 3763.

16 S. O. H. Gutschke, M. Molinier, A. K. Powell, R. E. P. Winpenny and P. T. Wood, Chem. Commun., 1996, 823.

17 J. Huheey, E. A. Keiter and R. L. Keiter, Inorganic Chemistry: Principles of Structure and Reactivity, 4th edn, Pearson Education, 2000 .

18 T. Moriya, Phys. Rev., 1960, 120, 91. 